\title{
Fragmentation spectra of strange quark matter in a type-II supernova scenario
}

\author{
Laura Paulucci*i \\ Instituto de Física - Universidade de São Paulo - Brazil \\ E-mail: paulucci@fma.if.usp.br
}

\section{Jorge E. Horvath}

Instituto de Astronomia, Geofísica e Ciências Atmosféricas - Universidade de São Paulo - Brazil E-mail: fotoneastro.iag.usp.br

\section{Frederique Grassi}

Instituto de Física - Universidade de São Paulo - Brazil

E-mail: grassiefma.if.usp.br

\begin{abstract}
We present and discuss calculations of strange quark matter fragmentation expected in the ejection of matter by a type-II supernova in the proposed scenario where the collapse of the progenitor is driven by the nuclear matter - quark matter conversion. Relevant issues such as the dynamical evolution of the ejecta and interactions with the outgoing stellar shells are addressed with the aim of understanding the mass distribution and spectra of strangelets at the sources.
\end{abstract}

International Symposium on Nuclear Astrophysics - Nuclei in the Cosmos - IX

25-30 June 2006

CERN

\footnotetext{
${ }^{*}$ Speaker.

$\dagger$ This work is supported by Fundação de Amparo à Pesquisa do Estado de São Paulo.
} 


\section{Introduction}

It has been realized [1] that systems composed of a confined Fermi-gas of up, down and strange quarks (strange quark matter) could have even a lower energy per baryon than iron, thus being absolutely stable. If strange quark matter (SQM) is indeed the ground state of cold baryonic matter there could be some important astrophysical implications. This hypothetical state could be created by weak interactions introducing the massive $s$ quark, if the "energy cost" of the mass is compensated by the availability of a new Fermi sea associated with this extra flavor, which lowers the Fermi energy of the $u$ and $d$ quark seas.

For the case of finite size lumps of SQM, (termed strangelets) a few terms have to be added to the bulk one in the free energy. Large lumps will have essentially the same number of quarks of bulk matter, with a small depletion of the massive strange quark resulting in a net positive charge, a feature also expected for small chunks [2],[3] which thus resemble heavy nuclei.

Considering the existence of strangelets among cosmic ray primaries, a few injection scenarios have been considered. The merging of compact stars (though not addressed in detail yet) [3] and strange matter formation in type II supernova are considered as likely sites.

Those particles could reach the earth and be detected either by land based experiments or in the magnetosphere [4]. In fact, there are claims of possible identification of these exotic cosmic rays (mainly the Centauro events, the HECRO-81 experiment [5], and more recently data from AMS-01 [6]) which present features such as their high penetration in the atmosphere, low charge-to-mass ratio and exotic secondaries.

\section{Strange quark matter in type-II supernova}

A model of type-II supernova explosion driven by nuclear matter $\rightarrow$ strange quark matter conversion in the inner shell of a collapsing massive star has been proposed [7] facing the still controversial results $[8]$ of the two currently favored models proposed to explain type-II supernova explosion: the prompt-shock and the delayed-shock (neutrino heating) mechanisms [9].

At the moment of bounce we have, according to current simulations, a central density of (23) $\rho_{0}$ inside the proto-neutron star. While this object is cooling by neutrino emission it suffers a substantial contraction. Assuming the quoted neutrino emission avoids a recollapse but is unable to produce the ejection of the outer layers of the star, the core of the proto-neutron star reaches densities for which it is conceivable that a high-strangeness quark-gluon plasma could appear.

Once the SQM is formed in the center of the star, the nuclear matter around it will soon be converted because of the lower energy of the quark matter state (by hypothesis) and this conversion will proceed outwards, likely in a detonation mode [7] but quite fast in any case. This mechanism may provide enough energy in the form of a detonation wave to sweep off the mantle and envelope of a massive star (an alternative ejection mechanism may be provided by the neutrino flux the from SQM at higher temperature). The matter ejected in this second shock would carry a contamination of strangelets due to turbulent mixing effects [10]. These ejection is the subject of our interest.

\subsection{Dynamics of the ejecta expansion}

Considering a spherical shell of SQM expanding during the first stage after the supernova 
explosion (ejecta dominated stage). A simple estimation of its cooling rate due to the emission of neutrinos, assuming the liquid phase is only supported for densities above the minimum density $n_{l i q} \geq 4 B$ we have determined a time after the explosion (of order $10^{-4} s$ ) and temperature at which the SQM will start to fragment (in the range of a few MeV). The problem is now how will this fragmentation proceed and, if possible, to calculate the particle (strangelet) spectra.

\subsection{Modeling the fragmentation}

The Statistical Multifragmentation Model (SMM) has been developed in the last decades [11] to deal with aspects related to the nuclear matter phase transition and nuclear multifragmentation. In face of these successful achievements, we have decided to apply the same method to describe the fragmentation of strange matter from the liquid phase (SQM in bulk) to the gaseous phase (represented by the strangelets).

We will base our analysis on the simplified version of the SMM presented by Bugaev et al. [12] where the system is analyzed in the grand canonical ensemble and afterwards taken to the thermodynamic limit, leading to analytical solutions.

The system is composed of many different fragments and is characterized by the total number of nucleons $A$, volume $V$ and temperature $T$. The system states are specified by the multiplicities $n_{a}\left(n_{a}=0,1,2 \ldots\right)$ of fragments of baryonic number $a$.

The partition function of a fragment with A nucleons is assumed to have the standard form [11]

$$
\omega_{a}=V\left(\frac{M T}{2 \pi}\right)^{3 / 2} z_{a}, \quad z_{a}=\exp \left(\frac{-f_{a}}{T}\right)
$$

where $M$ is the mass of the strangelet, $z_{a}$ is the fragment's intrinsic partition function $\left(z_{1}=4\right)$ and $f_{a}$ is the free internal energy of the fragment with baryon number $a$.

The grand canonical partition function is given by

$$
\mathscr{Z}(V, T, \mu)=\sum_{\left\{n_{a}\right\}} \prod_{a} \frac{1}{n_{a} !}\left[\left(V-b \sum_{a} a n_{a}\right) \frac{\omega_{a}(T, V)}{V} e^{\mu a / T}\right]^{n_{a}} \times \Theta\left(V-b \sum_{a} a n_{a}\right)
$$

where $b$ is the excluded volume and $\Theta$ is the Heaviside function.

A Laplace transformation of the grand canonical partition function can be performed in order to eliminate the Heaviside function and find the singularities of this function with which the gaseous and liquid phases are associated. This leads to the following expressions:

$$
\begin{gathered}
p_{g}(T, \mu)=T\left(\frac{m T}{2 \pi}\right)^{3 / 2}\left\{z_{1} e^{\frac{\mu-b p_{g}}{T}}+\sum_{a=2}^{\infty} a^{3 / 2} e^{\left[\left(\mu+W-b p_{g}\right) a-\sigma a^{2 / 3}-C a^{1 / 3}\right] / T}\right\} \\
p_{l}(T, \mu)=\frac{\mu+W}{b}
\end{gathered}
$$

$W, \sigma$ and $C$ being the internal bulk free energy, surface energy and curvature energy of the $a$ fragment, respectively, and $m$ is the strangelet's rest mass taken to be $930 \mathrm{MeV}$. 


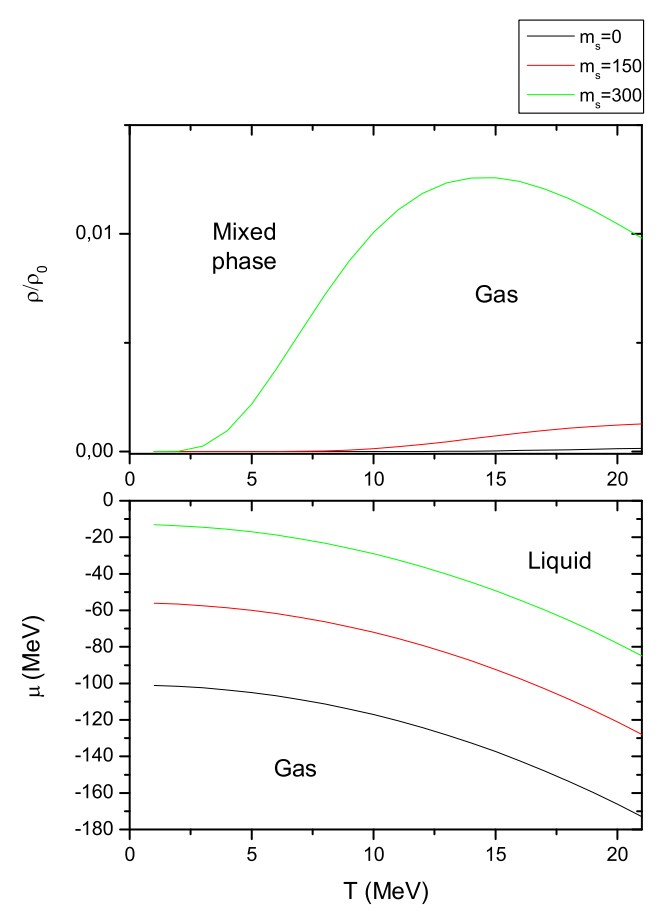

Figure 1: On the left panel we present the phase diagram in the $T \times \rho$ (above) and $T \times \mu$ (below) planes for different values of $m_{s}$. The liquid phase represents densities above $(2-3) \rho_{0}$. On the right, the atomic number where the fragment distribution function reaches its maximum in the mixed phase state for different values of $m_{s}$ (in units of $\mathrm{MeV}$ ).

The fragment distribution density can be obtained by differentiating the pressure $p_{g}$ with respect to the chemical potential, $\mu_{a}=a \mu$ (assuming chemical equilibrium)

$$
\mathscr{P}_{g}(a)=\frac{\partial}{\partial \mu_{a}} p_{g}=\left(\frac{m T}{2 \pi}\right)^{3 / 2} a^{3 / 2} e^{\left[\left(\mu+W-b p_{g}\right) a-\sigma a^{2 / 3}-C a^{1 / 3}\right] / T}
$$

We have constructed the phase diagram and derived the atomic number where the fragment distribution function reaches its maximum in the mixed phase state. The results found are very far from what one would expect for they show strangelets fragmenting for values of $\mathrm{A}$ which are (formally) much lower than 1 . We believe this means that the formalism is not adequate for the analysis of strange quark matter fragmentation, most probably indicating that it will fragment into few large chunks of matter, as advanced in previous works. Of course, if this is the case a small number of fragments cannot be studied using the statistical multifragmentation approach.

\section{Summary}

There are still some important features which were not considered on the calculations, for example, the dependence of the surface tension on the temperature. Besides that, the model considered does not employ a proper quantum treatment for the system, something that may lead to 
an improper analysis of the SQM phase transition. Further investigations changing the analysis method will probably tell if the statistical multifragmentation model is not adequate for obtaining the strangelet spectra or if the strange quark matter ejected will all decay to normal nuclear matter.

Other points that still need to be elucidated are the chemical equilibrium of strange quark matter through the process of expansion and cooling and if the fragments will decay to normal nuclear matter or remain as strangelets after fragmentation.

The fragmentation spectra as well as a proper treatment of the dynamic evolution of the fragments and its influence on the phase transition are still needed. Only then it will be possible to proceed the calculation on spallation of the ejected strangelets (if stable) with the expanding oxygen shell, relate the results with the problem of propagation through the interstellar medium and ultimately predict the contribution to the putative flux of strangelets that can be probed by several experiments.

\section{References}

[1] E. Witten, Phys. Rev. D 30, 272 (1984); A. Bodmer, Phys. Rev. D4, 1601 (1971); S. A. Chin and A. Kerman, Phys. Rev. Lett. 43, 1292 (1979)

[2] E. P. Gilson and R.L. Jaffe, Phys. Rev. Lett. 71, 332 (1993).

[3] J. Madsen, J. Physics G28, 1737 (2002)

[4] H. C. Rosu, hep-ph/9410029 (1994);

[5] J. D. Bjorken and L. McLerran, Phys. Rev. D20, 2353 (1979); M. Rybczynski, Z. Wlodarczyk and G. Wilk, hep-ph/0109225 (2001); T. Saito et al., Phys. Rev. Lett. 65, 2094 (1990)

[6] Choutko, V. (for the AMS-01 Collaboration), In : Kajita, T., Asaoka, Y., Kawachi, A., Matsubara Y. and Sasaki, M. (eds.) Proceedings of the 28th International Cosmic Ray Conference, Tsukuba, Japan, July 31- August 7, 1765 Universal Academic Press, Tokyo (2003)

[7] O. G. Benvenuto and J. E. Horvath, Phys. Rev. Lett. 63, 716 (1989); O. G. Benvenuto, J. E. Horvath and H. Vucetich, Int. J. Mod. Phys. A4, 257 (1989), 6, 4769 (1991)

[8] R. Buras et al., Phys. Rev. Lett., 90, 241101 (2003)

[9] H. A. Bethe, Rev. Mod. Phys. 62, 801 (1990) and references therein.

[10] O. G. Benvenuto and J. E. Horvath, Mod. Phys. Lett. A4, 1085 (1989)

[11] J. P. Bondorf et al., Phys. Rep. 257, 131 (1995) and references therein

[12] K. A. Bugaev et al., Phys. Rev. C62, 044320 (2000) 